\title{
Entwicklungsneurobiologie
}

\section{Grundlagen und Mechanismen der Neocortex-Expansion}

MICHAEL HEIDE, WIELAND B. HUTTNER

MAX-PLANCK-INSTITUT FÜR MOLEKULARE ZELLBIOLOGIE UND GENETIK, DRESDEN

\section{During primate, and notably human, evolution, the neocortex}

increased massively in size. This increase forms the basis of our higher cognitive abilities in comparison to other mammals. In recent years, major advances in understanding this evolutionary expansion have been achieved. Here, we would like to discuss the genetic foundation, principles and mechanisms underlying neocortical expansion.

DOI: $10.1007 / \mathrm{s} 12268-020-1411-5$

(C) Die Autoren 2020

Die Expansion des Neocortex während der Evolution des Menschen bildet eine Grundlage unserer - im Vergleich zu anderen Primaten - höheren kognitiven Fähigkeiten. Im Vergleich zu unserem nächsten noch lebenden Verwandten, dem Schimpansen, hat sich die Größe des Neocortex verdreifacht. Die Ursache dieser massiven VergröBerung liegt in der Aktivität und dem Verhalten neuraler Stamm- und Vorläuferzellen, die die Neurone des sich entwickelnden $\mathrm{Neo}^{-}$ cortex bilden. Diese Zellen befinden sich sowohl in Säugetieren mit einem kleinen, ungefalteten Neocortex (wie bei der Maus) als auch in Säugetieren mit einem großen, gefalteten Neocortex (wie beim Menschen) in

zwei Keimzonen (Abb. 1). Die erste liegt apikal, direkt den Ventrikel begrenzend, und ist die ventrikuläre Zone (VZ), die hauptsächlich die Zellkörper der apikalen radialen Gliazellen (aRG) beherbergt. Basal zu dieser Zone befindet sich die subventrikuläre Zone (SVZ), die die Zellkörper der basalen Vorläuferzellen (basal progenitors, BPs) beherbergt. In der Maus sind dies hauptsächlich basale intermediäre Vorläuferzellen und sehr wenige basale radiale Gliazellen (bRG). Im Menschen ist die SVZ stark vergrößert, und es kann eine innere und eine äußere SVZ unterschieden werden. Im Gegensatz zur Maus enthält die humane SVZ einen wesentlich höheren Anteil an bRG (Abb. 1). Von daher

wird davon ausgegangen, dass diese Zellen eine Schlüsselrolle in der Expansion des Neocortex ausüben [1].

\section{Genetische Grundlagen der Neocortex-Expansion}

Wesentliche Grundlage der NeocortexExpansion bildet die Information, die in der DNA codiert ist. Während der Evolution kam es zu Mutationen der DNA, die zur Expansion des Neocortex beitrugen. Diese Mutationen reichen von einzelnen Nukleotiden bis hin zu Duplikationen kompletter Gensegmente. Des Weiteren können die Mutationen unterschiedliche Bereiche des Genoms betreffen.

Zum einen können sie regulatorische Regionen, wie z. B. Promotoren, betreffen, wodurch die Expression der betreffenden Gene beeinflusst werden kann (Abb. 2A). Zum anderen können diese Mutationen in codierenden Regionen auftreten (Abb. 2B); dabei kann beispielsweise die Aminosäuresequenz eines Proteins und somit dessen Funktion bzw. Lokalisierung verändert werden (Abb. 2B, [2]). Diese kleineren Mutationen können jedoch nicht nur zu einer Veränderung der Funktion des Proteins führen, sondern auch zu seinem Funktionsverlust bzw. sogar zu einem Verlust des funktionalen Gens (Abb. 2C). Eine verringerte Funktion des Proteins bzw. der Verlust des Gens kann, wenn dies einen Vorteil für den Organismus darstellt, selektioniert werden. Dabei sammeln solche Gene im Verlauf der Evolution Mutationen an, die schließlich zum Verlust des Gens führen, beispielsweise durch die Entstehung eines Stoppcodons kurz nach dem Translationsstart (Abb. 2C, [2]).

Im Gegensatz zu den beschriebenen kleinen Mutationen gibt es auch große, wie die Duplikation von Gensegmenten, die zu der Entste- 
A Veränderungen in regulatorischen Sequenzen
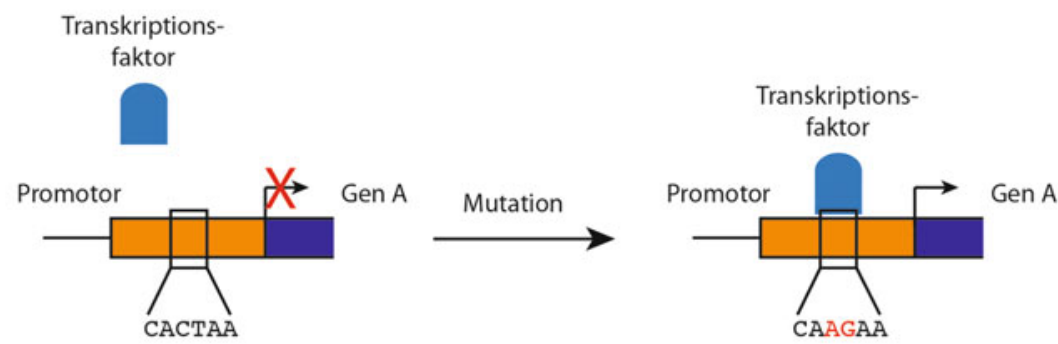

B Veränderungen in codierenden Sequenzen
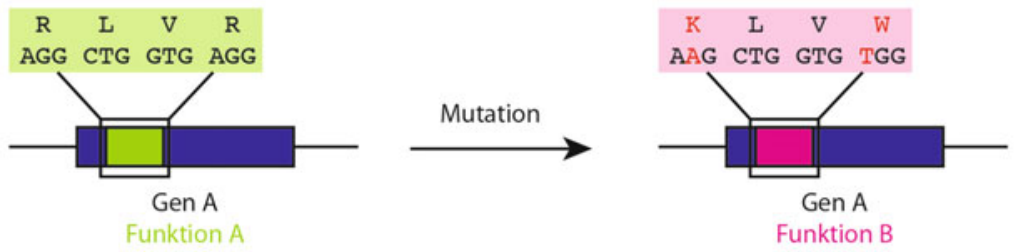

C Verlust von Genen
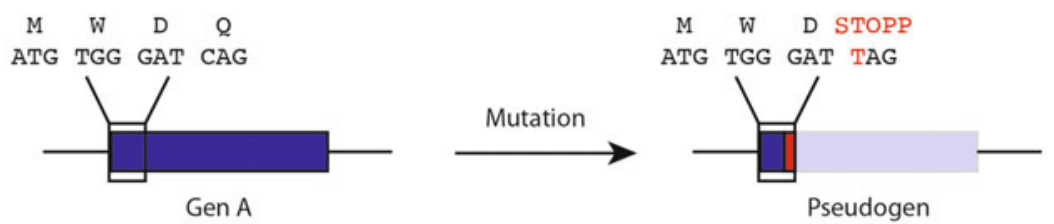

D Entstehung neuer Gene
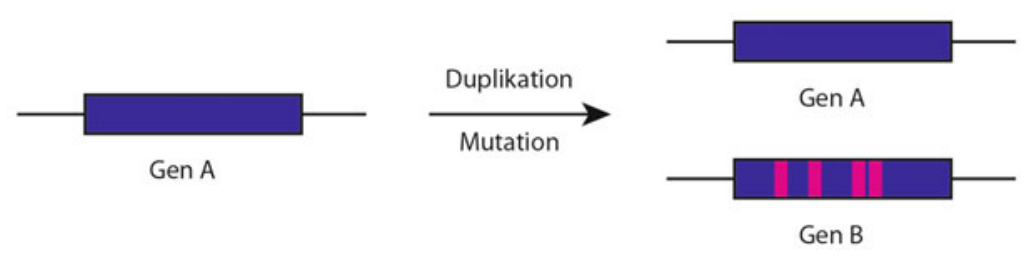

$\Delta$ Abb. 2: Genetische Grundlagen der Neocortex-Expansion. A, Veränderungen in regulatorischen Sequenzen. B, Veränderungen in codierenden Sequenzen. C, Verlust von Genen. D, Entstehung neuer Gene.

hung neuer Gene führen kann. Durch eine solche segmentale Duplikation werden Gene verdoppelt (Abb. 2D). Dies sind zunächst einfache Veränderungen der Kopienzahl eines Gens, die jedoch - wenn vorteilhaft - in der Population fixiert werden können. Die duplizierten Gene können dadurch beispielsweise unter die Kontrolle eines anderen Promotors geraten und so völlig anders exprimiert werden als das ursprüngliche Gen. Mutationen in duplizierten Genen können aber auch zu völlig neuen Funktionen führen (Abb. 2D, [2]).

Daten aus unserer Arbeitsgruppe, aber auch aus anderen Laboren, zeigen, dass solche neuen Gene einen entscheidenden Bei- trag zur Expansion des menschlichen Neocortex leisteten [3-6]. Hervorzuheben sind hier insbesondere jene Gene, die in der menschlichen Linie nach der Abspaltung der Linie, die zu Schimpansen und Bonobos führte, entstanden sind, die humanspezifischen Gene. Dies wird durch den oben beschriebenen Fakt unterstrichen, dass es nach der Abspaltung der menschlichen Linie zu einer Verdreifachung der Neocortex-Größe kam. Um diese Expansion zu verstehen, müssen insbesondere jene neocortikalen Vorläuferzellen untersucht werden, die in Säugetieren mit einem großen und gefalteten Neocortex vermehrt vorkommen, die oben erwähnten bRG (Abb. 1, [1]).
Vor einigen Jahren hat unser Labor eine vergleichende Transkriptomanalyse von aRG, bRG und Neuronen des Menschen und der Maus durchgeführt und konnte ein humanspezifisches Gen identifizieren, das bevorzugt in aRG und bRG exprimiert ist, ARHGAP11B [3]. Des Weiteren konnte gezeigt werden, dass dieses Gen, wenn man es im Neocortex von Mausembryonen exprimiert, zu einer Vermehrung der BPs und in 50 Prozent der Fälle zu einer Faltung des normalerweise ungefalteten Mausneocortex führt [3]. Somit konnte mit $A R H G A P 11 B$ ein humanspezifisches Gen identifiziert werden, das eine Schlüsselrolle in der humanen Neocortex-Expansion einnimmt. Aufbauend auf dieser Studie identifizierten wir 14 weitere humanspezifische und 35 primatenspezifische, aber nicht-humanspezifische Gene, die eine bevorzugte Expression in neuralen Stamm- und Vorläuferzellen zeigten [4]. Darüber hinaus konnten wir und zwei andere Labore zeigen, dass eines dieser weiteren humanspezifischen Gene, NOTCH2NL, einen ähnlichen Effekt wie $A R H G A P 11 B$ aufweist [4-6].

\section{Prinzipien und Mechanismen der Neocortex-Expansion}

Generell lassen sich zwei grundlegende Prinzipien unterscheiden, die zu einer Vergrößerung des Neocortex führen können. Eines ist die Länge der neurogenen Phase, also die Zeit, in welcher Neurone produziert werden. Je länger diese Phase ist, desto mehr Neurone können produziert werden (Abb. 3A). In diesem Zusammenhang konnten mathematische Modellierungen zeigen, dass es zwei Klassen von Säugetieren gibt, solche mit einem nicht oder schwach gefalteten Neocortex und solche mit einem stark gefalteten Neocortex. Innerhalb jeder dieser Klassen lässt sich der Anstieg in der Zahl der Neurone zwischen verschiedenen Säugetierarten durch eine Zunahme der Länge der neurogenen Phase erklären [7]. Das zweite Prinzip betrifft die Größe der Vorläuferzellpopulation bzw. die proliferative Kapazität dieser Zellen. Je größer diese Population ist bzw. je stärker diese proliferiert, desto mehr Vorläuferzellen stehen zur Verfügung, die Neurone produzieren können (Abb. 3B, [1]).

Diesen beiden Prinzipien liegen unterschiedliche Mechanismen zugrunde, die zu einer Verlängerung der neurogenen Phase bzw. einer Vergrößerung der Vorläuferzellpopulation führen. Was Letztere betrifft, sollen hier drei unterschiedliche Mechanismen 
Hier steht eine Anzeige.

Springer 


\section{Prinzipien der Neokortex-Expansion}

A Länge der neurogenen Phase
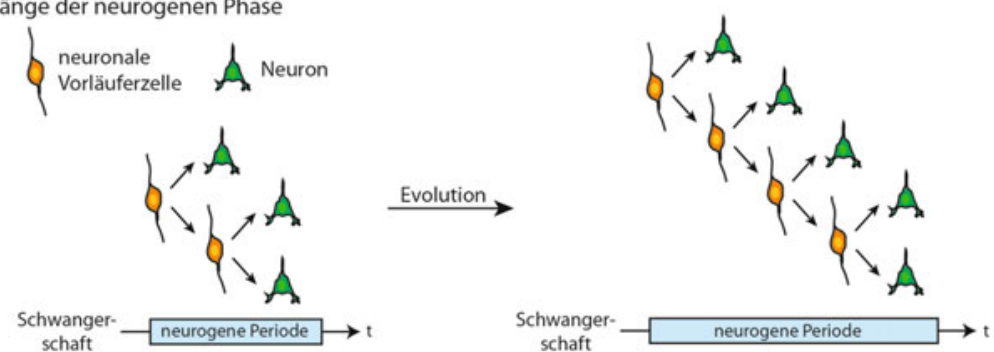

B Populationsgröße
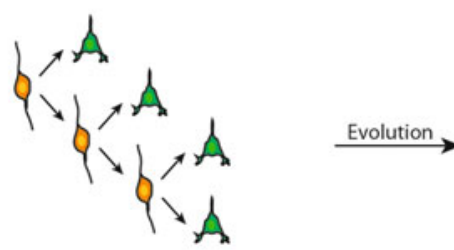

\section{Mechanismen der Neokortex-Expansion}

C Veränderung der Expression

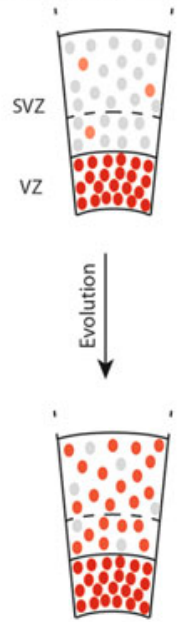

D Veränderung der Morphologie

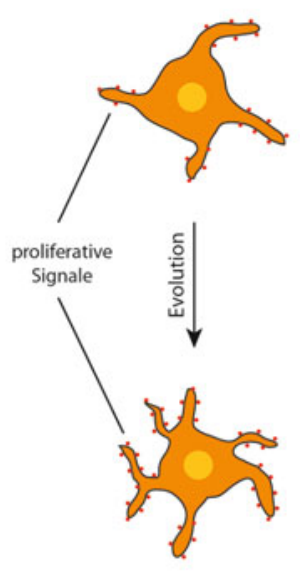

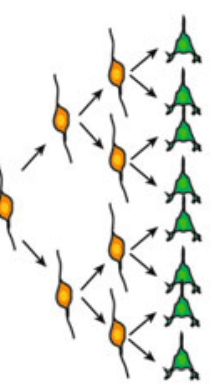

$\mathrm{E}$

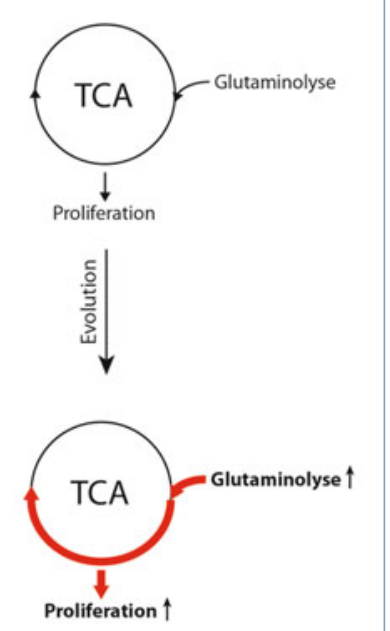

$\Delta$ Abb. 3: Prinzipien und Mechanismen der Neocortex-Expansion. A, Neocortex-Expansion durch eine Verlängerung der neurogenen Periode (von links nach rechts). Diese Verlängerung führt zu einer größeren Anzahl an Zellteilungen der neuronalen Vorläuferzellen (gelb) und somit zu einem Anstieg der Neuronenzahl (grün). B, Neocortex-Expansion durch eine Zunahme der Populationsgröße der neuronalen Vorläuferzellen (gelb) (von links nach rechts). Im Beispiel links ist die Populationsgröße der neuronalen Vorläuferzellen während der Neocortex-Entwicklung konstant, und Neurone (grün) werden durch wiederholte asymmetrische Zellteilungen der neuronalen Vorläuferzellen geboren, bis zur terminalen symmetrisch neurogenen Zellteilung. Im Beispiel rechts nimmt die Populationsgröße der neuronalen Vorläuferzellen (gelb) während der Neocortex-Entwicklung durch wiederholte symmetrisch proliferative Zellteilungen stetig zu, sodass die terminale symmetrisch neurogene Zellteilung dieser neuronalen Vorläuferzellen in einer erhöhten Neuronenzahl (grün) resultiert. C, Veränderung der Expression. SVZ: subventrikuläre Zone; VZ: ventrikuläre Zone. D, Veränderung der Morphologie. E, Veränderung des Metabolismus.

vorgestellt werden, von denen in den letzten Jahren gezeigt wurde, dass sie vermutlich einen erheblichen Beitrag zur NeocortexExpansion leisteten.

Ein relativ einfacher Mechanismus ist die Veränderung der Expression von vorhan- denen Genen (Abb. $\mathbf{3 C})$. Dies ist insbesondere effektiv, wenn Gene betroffen sind, die die Identität bzw. Proliferation von Zellen beeinflussen. So ist beispielsweise der Transkriptionsfaktor Pax6 in BPs der Maus sehr schwach bzw. nicht exprimiert, in BPs von
Primaten ist Pax6 jedoch relativ stark exprimiert [8]. Eine anhaltende selektive Expression von Pax6 in aRG und den von diesen gebildeten BPs führt zu einer Vermehrung der BPs und einer verstärkten Produktion von Neuronen der oberen cortikalen Schichten [9]. Somit reicht die veränderte Expression eines einzelnen Transkriptionsfaktors aus, um eine den Primaten ähnliche Vermehrung von BPs zu erreichen.

Es entstanden während der Evolution jedoch auch weitaus innovativere Mechanismen als die simple Veränderung der Genexpression. So konnte unser Labor vor Kurzem zeigen, dass die Veränderung der Morphologie der Vorläuferzellen vermutlich einen entscheidenden Beitrag zur NeocortexExpansion leistete (Abb. 3D). Demnach führte die Expression von menschlichem, membrangebundenem PALMDELPHIN $(P A L M D)$ zu einer Vermehrung der BP-Fortsätze und einer verstärkten Proliferation der BPs in der Maus und dem Frettchen. Hingegen führt ein CRISPR/Cas9-vermittelter Knock-out von PALMD im fötalen menschlichen Neocortex zu einer Verringerung der Anzahl an Zellfortsätzen und damit einhergehend zu einer verringerten BP-Proliferation [10]. Diese Vermehrung an Zellfortsätzen erlaubt es den BPs vermutlich, vermehrt proliferative Signale aus der Umgebung aufzunehmen und sich dadurch verstärkt zu vermehren (Abb. 3D).

Stark proliferierende Zellen, wie z. B. Tumorzellen, zeigen einen veränderten Metabolismus [11]. Dies wirft die Frage auf, ob ein veränderter Metabolismus ein weiterer Mechanismus der Neocortex-Expansion sein könnte (Abb. 3E). Tatsächlich konnte unser Labor in einer kürzlich veröffentlichten Studie zeigen, dass ein veränderter Metabolismus von Vorläuferzellen ein solcher Schlüsselmechanismus sein kann [12]. So konnte gezeigt werden, dass das oben erwähnte humanspezifische Gen ARHGAP11B - im Gegensatz zum ursprünglichen Gen $A R H$ GAP11A - in Mitochondrien importiert wird. Dort interagiert es mit dem Adenin-Nukleotid-Translokator (ANT) und inhibiert so die mitochondrial permeability transition pore, die von ANT reguliert wird. Dies resultiert in verstärkter Glutaminolyse in BPs und führt letzten Endes zu einer Vermehrung dieser Zellen (Abb. 3E, [12]).

\section{Fazit}

Zusammengefasst konnte in den vergangenen Jahren eine Reihe von Veränderungen 
im menschlichen Genom identifiziert werden, die diverse zelluläre Mechanismen dahingehend modulieren, dass sich die Population und Teilungsaktivität der relevanten Vorläuferzellen im sich entwickelnden Neocortex vergrößern. Dadurch werden mehr Neurone, insbesondere für die oberen cortikalen Schichten, gebildet, was letztendlich in einer Vergrößerung der Großhirnrinde resultiert.

\section{Literatur}

[1] Florio M, Huttner WB (2014) Neural progenitors, neurogenesis and the evolution of the neocortex. Development 141:2182-2194

[2] Heide M, Long KR, Huttner WB (2017) Novel gene function and regulation in neocortex expansion. Curr Opin Cell Biol 49:22-30

[3] Florio M, Albert M, Taverna E et al. (2015) Human-specific gene $A R H G A P 11 B$ promotes basal progenitor amplification and neocortex expansion. Science 347:1465-1470

[4] Florio M, Heide M, Pinson A et al. (2018) Evolution and cell-type specificity of human-specific genes preferentially expressed in progenitors of fetal neocortex. eLife 7:e32332 [5] Suzuki IK, Gacquer D, Van Heurck R et al. (2018) Humanspecific NOTCH2NL genes expand cortical neurogenesis through Delta/Notch regulation. Cell 173:1370-1384 [6] Fiddes IT, Lodewijk GA, Mooring M et al. (2018) Humanspecific NOTCH2NL genes affect notch signaling and cortical neurogenesis. Cell 173:1356-1369

[7] Lewitus E, Kelava I, Kalinka AT et al. (2014) An adaptive threshold in mammalian neocortical evolution. PLoS Biol 12:e1002000

[8] Fish JL, Kennedy H, Dehay C et al. (2008) Making bigger brains - the evolution of neural-progenitor-cell division. J Cell Sci 121:2783-2793

[9] Wong FK, Fei JF, Mora-Bermudez F et al. (2015) Sustained Pax6 expression generates primate-like basal radial glia in developing mouse neocortex. PLoS Biol 13:e1002217

[10] Kalebic N, Gilardi C, Stepien B et al. (2019) Neocortical expansion due to increased proliferation of basal progenitors is linked to changes in their morphology. Cell Stem Cell 24:535-550

[11] Yang L, Venneti S, Nagrath D (2017) Glutaminolysis: a hallmark of cancer metabolism. Annu Rev Biomed Eng 19:163-194

[12] Namba T, Doczi J, Pinson A et al. (2020) Human-specific $A R H G A P 11 B$ acts in mitochondria to expand neocortical progenitors by glutaminolysis. Neuron 105:867-881

Funding: Open Access funding provided by Projekt DEAL.

Open Access: Dieser Artikel wird unter der Creative Commons Namensnennung 4.0 International Lizenz veröffentlicht, welche die Nutzung, Vervielfältigun erlaubt, sofern Sie den/die ursprünglichen Autor(en) und die Quelle ordnungsgemäß nennen, einen Link zur Creative Commons Lizenz beifügen und angeben, ob Änderungen vorgenommen wurden. Die in diesem Artikel enthaltenen Bilder und sonstiges Drittmaterial unterliegen ebenfalls der genannten Creative Commons Lizenz, sofern sich aus der Abbildungslegende nichts anderes ergibt. Sofern das betreffende Material nicht unter der genannten Creative Commons Lizenz steht und die betreffende Handlung nich nach gesetzlichen Vorschriften erlaubt ist, ist für die oben aufgeführte Weiterverwendungen des Materials die Einwilligung des jeweiligen

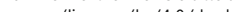

Michael Heide
2005-2009 Biologiestudium an der Universität Tübingen, dort 2009-2010 Diplom-
arbeit am Anatomischen Institut. 2010-2014 Promotion am Institut für Anatomie und
Zellbiologie der Universität Heidelberg. Seit 2015 Postdoktorand am Max-Planck-
Institut für molekulare Zellbiologie und Genetik, Dresden.

Hier steht eine Anzeige. Springer
Korrespondenzadresse:

Dr. Michael Heide

Prof. Dr. Wieland B. Huttner

Max-Planck-Institut für molekulare Zellbiologie und Genetik

Pfotenhauerstraße 108

D-01307 Dresden

heide@mpi-cbg.de

huttner@mpi-cbg.de 